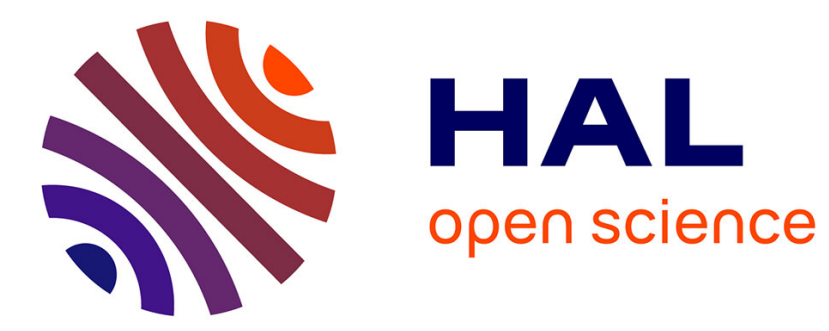

\title{
LiNbO3 Mach-Zehnder modulator with chirp adjusted by ferroelectric domain inversion
}

N. Courjal, H. Porte, A. Martinez, Jp Goedgebuer

\section{To cite this version:}

N. Courjal, H. Porte, A. Martinez, Jp Goedgebuer. LiNbO3 Mach-Zehnder modulator with chirp adjusted by ferroelectric domain inversion. IEEE Photonics Technology Letters, 2002, 14 (11), pp.15091511. 10.1109/LPT.2002.803365 . hal-00093745

\section{HAL Id: hal-00093745 \\ https://hal.science/hal-00093745}

Submitted on 19 Apr 2021

HAL is a multi-disciplinary open access archive for the deposit and dissemination of scientific research documents, whether they are published or not. The documents may come from teaching and research institutions in France or abroad, or from public or private research centers.
L'archive ouverte pluridisciplinaire HAL, est destinée au dépôt et à la diffusion de documents scientifiques de niveau recherche, publiés ou non, émanant des établissements d'enseignement et de recherche français ou étrangers, des laboratoires publics ou privés. 


\title{
$\mathrm{LiNbO}_{3}$ Mach-Zehnder Modulator With Chirp Adjusted by Ferroelectric Domain Inversion
}

\author{
Nadege Courjal, Henri Porte, Anthony Martinez, and Jean-Pierre Goedgebuer
}

\begin{abstract}
Domain inversion under coplanar waveguide electrodes is proposed to improve the frequency-chirping behavior of z-cut $\mathrm{LiNbO}_{3}$ Mach-Zehnder modulators. This is achieved by introducing phase reversal electrode section in tandem with inverted ferroelectric domain section. The resulting chirp is shown to be related to the length of the inverted domain. The method opens the way to single-drive modulators with predetermined chirp parameter. The fabrication of such modulators is described, and experimental results confirm that the $\alpha$-chirp parameter can be more than ten times smaller than that of a conventional Z-cut device.
\end{abstract}

Index Terms-Chirp modulation, domain inversion, electrooptic modulation, ferroelectric, lithium niobate.

\section{INTRODUCTION}

D ISPERSION of short optical pulses propagating in optical fibers is one of the main limiting factors for high bit rate transmission. When the pulses are produced by a chirped source like a directly modulated semiconductor laser, this effect may even be worse. This explains why external modulation is usually preferable than direct modulation in $1.55 \mu \mathrm{m}$ telecommunication systems. $\mathrm{LiNbO}_{3}$ Mach-Zehnder modulators (MZMs) have known an increasing interest, because their negligibly small wavelength dependence makes them suitable for both time division multiplexing [1] and wavelength division multiplexing optical transmission systems [2].

$\mathrm{Z}$-cut $\mathrm{LiNbO}_{3}$ modulators seem to be more promising devices than $\mathrm{X}$-cut $\mathrm{LiNbO}_{3}$ modulators, because they can provide a larger bandwidth and a lower driving voltage, particularly if the structure is ridged [3]. In contrast to X-cut modulators [4], Z-cut devices exhibit yet a nonzero chirp parameter (typically $\alpha \approx-0.7$ ) that cannot be easily settled, unless they are designed with a dual drive topology [5]. This situation is due to the push-pull configuration of the coplanar waveguide (CPW) electrodes, which introduces an asymmetry between the two arms of the MZM, the waveguide aligned under the hot line of the CPW electrode being submitted to a higher electrooptic efficiency than that placed under one of the lateral ground plane electrodes.

In this letter, we report a new single-drive Z-cut modulator scheme, which provides the feasibility of setting the chirp parameter to zero or to another determined value.

N. Courjal, A. Martinez, and J.-P. Goedgebuer are with the Laboratoire d'Optique P. M. Duffieux, Université de Franche Comté, Institut des Microtechniques (IMFC), 25030 Besançon Cedex, France (e-mail: nadege.bodin@univfcomte.fr).

H. Porte is with the Photline Technologies, 25001 Besançon Cedex 3, BP71025, France.

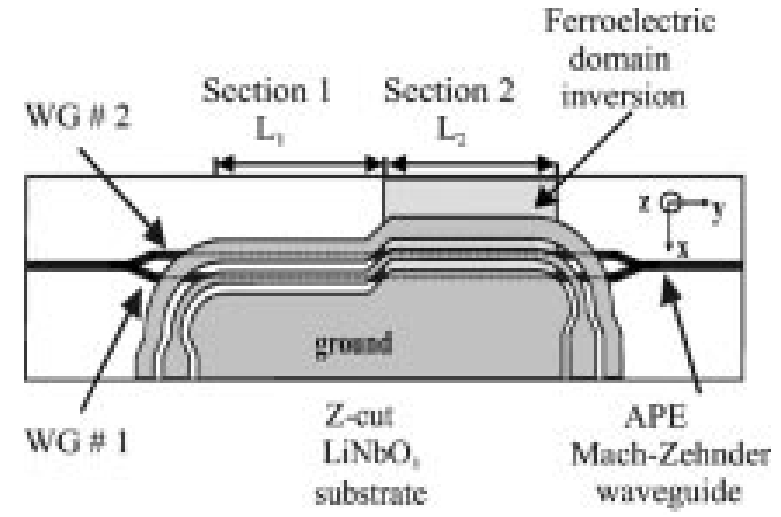

Fig. 1. Structure of a z-cut MZM with inverted domain.

\section{DESIGN}

In a standard electrooptic Z-cut MZM, the chirp parameter is known to be expressed as a function that depends only on the overlap coefficients of each arm of the Mach-Zehnder at a small amplitude signal and quadrature bias. Due to the asymmetry previously mentioned, this standard chirp parameter $\alpha_{S}$ can be expressed as

$$
\alpha_{s}=\frac{\eta_{G}+\eta_{C}}{\eta_{G}-\eta_{C}}
$$

where $\eta_{C}$ and $\eta_{G}$ are the overlap coefficients between the electric field and the optical field under the central electrode and the ground electrodes, respectively. In what follows, we propose to divide the standard structure into two sections with opposite ferroelectric domain signs. A waveguide of the MZM is, thus, covered by the signal electrode in one section and by the ground electrode in the other section. This scheme is shown to yield a chirp parameter that can be adjusted to zero or to any desired value.

The device is depicted in Fig. 1. It consists of a symmetrical Mach-Zehnder of length $L$, with the same waveguide widths for both branches. The substrate is a Z-cut lithium niobate crystal. A section of length $L_{2}$ of the waveguide branches is processed to have a ferroelectric domain inversion whereas the orientation of the first section of the waveguide arms of length $L_{1}=L-L_{2}$ remains unchanged. The electrooptic coefficient $r_{33}$ is assumed to be positive in the noninverted section, and negative in the inverted section. A set of coplanar electrodes is patterned over the waveguide arms. In section \#1, which is the noninverted ferroelectric domain section, the central line of the electrode is centered over waveguide \#1, yielding an overlap coefficient $\eta_{C}$, while the edge of one lateral ground electrode is aligned along the edge of waveguide \#2, yielding an overlap coefficient 


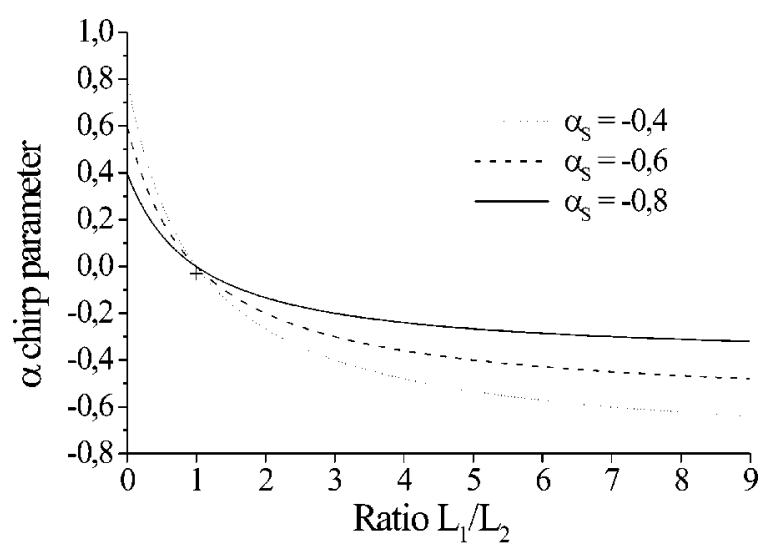

Fig. 2. $\alpha$ chirp parameter of the structure versus $L_{1} / L_{2}$ for a modulator with lossless electrodes $\alpha_{S}$ are the chirp parameters of standard modulators with the same transmission lines. The cross (+) stand for experimental measurement of the chirp at the 2.5-GHz driving frequency and for $L_{1}=L_{2}$.

Conversely, in section \#2, which is the inverted region, the central line is centered over waveguide \#2, while the second ground electrode is aligned along the edge of waveguide \#1. A voltage $V_{z}$ is applied to the electrodes. Due to the inversion of both the crystal ferroelectric orientation domain and the electrical field, the optical phase of a light beam of wavelength $\lambda$ after passing through each of the arms of the MZM can be expressed as, respectively

$$
\begin{aligned}
& \varphi_{1}=K \cdot V_{z} \cdot\left(\gamma_{1} \cdot \eta_{C} \cdot L_{1}-\gamma_{2} \cdot \eta_{G} \cdot L_{2}\right) \\
& \varphi_{2}=K \cdot V_{z} \cdot\left(\gamma_{1} \cdot \eta_{G} \cdot L_{1}-\gamma_{2} \cdot \eta_{C} \cdot L_{2}\right)
\end{aligned}
$$

where $\gamma_{1}$ and $\gamma_{2}$ account for the lossy transmission of the electrical signal through the optical path in region 1 and 2, respectively [see (5) and (6) for more details]. $K=\pi n_{e}^{3}\left|r_{33}\right| / \lambda g$ is a constant related to the extraordinary refractive index $n_{e}$ of lithium niobate and to the electrode gap $g$. For simplicity, (2) and (3) have been derived assuming the electrical index is matched to the optical refractive index, which is a condition required in high-speed modulators. It should be noted that the optical phase difference $\left(\varphi_{1}-\varphi_{2}\right)$ at the output of the Mach-Zehnder interferometer is identical to the phase difference $\left(\varphi_{1}-\varphi_{2}\right)_{s}$ at the output of a standard MZM, i.e., a modulator with CPW electrodes of same length $L_{1}+L_{2}$ and with no inverted region.

The interest of this new configuration lies in the chirp parameter $\alpha$ [6], which takes the form of

$$
\alpha=\frac{\left(\gamma_{1} L_{1}-\gamma_{2} L_{2}\right)}{\left(\gamma_{1} L_{1}+\gamma_{2} L_{2}\right)} \times \frac{\left(\eta_{C}+\eta_{G}\right)}{\left(\eta_{C}-\eta_{G}\right)}=\frac{\left(\gamma_{1} L_{1}-\gamma_{2} L_{2}\right)}{\left(\gamma_{1} L_{1}+\gamma_{2} L_{2}\right)} \times \alpha_{S} .
$$

The right factor in (4) is the $\alpha_{s}$ chirp parameter of a standard Z-cut modulator, expressed in (1), while the left term is typical of the presented structure. Since $\eta_{C}$ is significantly different from $\eta_{G}$, it is clear from (4) that the structure enables one to control the chirping behavior of the modulator by simply adjusting the ratio $L_{1} / L_{2}$.

Let us first consider the case of a modulator with lossless electrodes, i.e., $\gamma_{1}=\gamma_{2}=1$. Then, we have no chirp when $L_{1}=L_{2}$. If $L_{1} / L_{2} \neq 1$, the $\alpha$-parameter depends on $\alpha_{S}$, which is determined by the lateral geometry of the electrodes. Fig. 2 displays the values of $\alpha$ that can be obtained by varying $L_{1} / L_{2}$

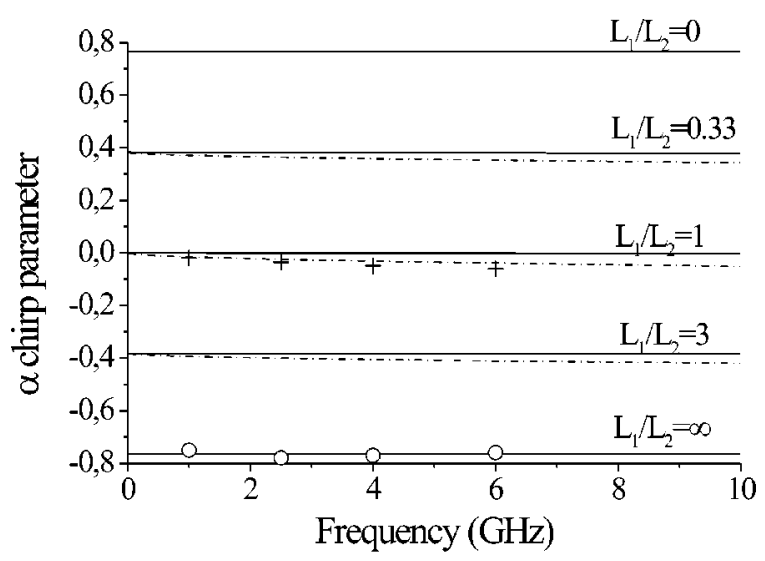

Fig. 3. Frequency chirping performance of a modulator with (dashed lines) and without (solid lines) electrical losses. The curves were plotted for a modulator with the following parameters. Total active length: $L_{1}+L_{2}=$ $1.7 \mathrm{~cm} ; \alpha_{S}$ of the corresponding standard modulator: -0.77 ; Electrical absorption: $0,4 \mathrm{~dB} / \mathrm{cm} \sqrt{\mathrm{GHz}}$. Crosses (+) and circles (o) stand for experimental measurement of the chirp at a driving frequency of $1,2.5,4$, and $6 \mathrm{GHz}$ for a modulator with identical sections lengths and a modulator with no inverted ferroelectric domain section, respectively.

for different values of $\alpha_{S} . L_{1} / L_{2}=0$ and $L_{1} / L_{2}=\infty$ are the two limits between which the $\alpha$-chirp parameter of the modulator can be adjusted. These limits would correspond to standard modulators designed with a single inverted or noninverted ferroelectric section, respectively.

In the case of high-speed modulation, losses of the microwave line cannot however be considered as negligibly small. Since the conductor losses are related to the square root of the frequency $f$ of the voltage through (5) and (6)

$$
\begin{aligned}
& \gamma_{1}=\frac{1}{L_{1}} \int_{0}^{L_{1}} \exp \left(-a_{0} f^{1 / 2} z\right) d z \\
& \gamma_{2}=\frac{1}{L_{2}} \int_{L_{1}}^{L_{2}} \exp \left(-a_{0} f^{1 / 2} z\right) d z
\end{aligned}
$$

it can be expected that the resulting chirp is also frequencydependent. Taking a typical loss coefficient $a_{0}=0.023 \mathrm{~Np} /$ $\mathrm{GHz}^{1 / 2} / \mathrm{cm}$ (equivalent to $0.2 \mathrm{~dB} / \mathrm{cm} / \mathrm{GHz}^{1 / 2}$ ), we have plotted in Fig. 3 the frequency-dependence of $\alpha$ over a $10-\mathrm{GHz}$ band for a modulator of total length $L=L_{1}+L_{2}=1.7 \mathrm{~cm}$ and for different values of $L_{1}$. The values of $\alpha$ were calculated from (4)-(6). Those values can be compared with the chirp parameter $\alpha_{S}=-0.8$ (which is quasi frequency-independent) of a standard modulator with the same active length $L=1.7 \mathrm{~cm}$.

Fig. 3 also shows that the dependence of the $\alpha$-chirp parameter upon electrical loss becomes increasingly important as the structure is symmetric. Nevertheless, in that case the difference between the $\alpha$ parameter for an electrical line with and without losses does not exceed 0.15 at $40 \mathrm{GHz}$, a value which is much lower than that of the corresponding standard structure. Therefore, the structure represents a significative improvement of the frequency chirping behavior for high speed $\mathrm{Z}$-cut $\mathrm{LiNbO}_{3}$ modulators.

Following these preliminary theoretical results, we have fabricated a technological process to realize a modulator with two sections of opposite ferroelectric signs in order to verify the modulation behavior and the chirp parameter of the structure. 


\section{EXPERIMENTAL RESULTS}

At first, the domain inversion was produced through a rectangular window obtained in a photoresist by photolithography on the $+\mathrm{z}$ face of a 0.5 -mm-thick single- $\mathrm{z}-\mathrm{cut} \mathrm{LiNbO}_{3}$ wafer. Domain inversion was performed using this window by applying a high voltage pulse of $22 \mathrm{kV} / \mathrm{mm}$ through liquid electrodes at room temperature. The total electric charge as a result of inversion was $Q=1258 \mu \mathrm{C}$, which is in good agreement with the theoretical value of $Q=2 P_{S} A=1248 \mu \mathrm{C}$ calculated from the spontaneous polarization $P_{S}=78 \mu \mathrm{C} / \mathrm{cm}^{2}$ of $\mathrm{LiNbO}_{3}$ and the area $A=2 \mathrm{~cm} \times 4 \mathrm{~cm}$ of the photoresist window. In a second step, a single-mode optical Mach-Zehnder waveguide was fabricated by annealed proton exchange. This step was realized through a $\mathrm{SiO}_{2}$ mask in benzoïc acid at $177^{\circ} \mathrm{C}$ for $3.5 \mathrm{~h}$. The process was followed with annealing of the optical waveguide at $330{ }^{\circ} \mathrm{C}$ for $10 \mathrm{~h}$. A silica buffer layer was then evaporated onto the sample surface with a thickness of about $1 \mu \mathrm{m}$. Finally 5- $\mu \mathrm{m}$-thick Au-coplanar electrodes were electrodeposited over the Mach-Zehnder waveguide.

The modulator exhibited two identical sections lengths $L_{1}=L_{2}=0.85 \mathrm{~cm} \mathrm{~A} \mathrm{standard} \mathrm{modulator} \mathrm{with} L=1.7 \mathrm{~cm}$ electrodes length and no inverted ferroelectric domain section $\left(L_{1} / L_{2}=\infty\right)$ was fabricated on the same wafer in view of comparing the electrooptical efficiencies and the chirping behaviors. The gap between electrodes was $g=30 \mu \mathrm{m}$ in both cases. The extinction ratio $R$ and the half-wave voltage $V_{\pi}$ were measured at low-frequency $(1 \mathrm{kHz})$ to be, respectively, $R=14 \mathrm{~dB}$, and $V_{\pi}=6.6 \mathrm{~V}$, which is in good agreement with the $R_{S}=16 \mathrm{~dB}$ extinction ratio and $V_{\pi S}=6.9 \mathrm{~V}$ half wave voltage evaluated from the standard modulator. A measure of the electrical characteristics lead to the electrical loss coefficient $a_{0}=0.10 \mathrm{~dB} / \mathrm{cm} / \mathrm{GHz}^{1 / 2}$. For this nonoptimized structure, the $-3 \mathrm{~dB}$ electrooptical bandwidth was assessed to be $4 \mathrm{GHz}$.

The chirp parameter was then evaluated at modulation frequencies of $1.0,2.5,4$, and $6 \mathrm{GHz}$ by optical spectral analysis of the signal with a high-resolution scanning Fabry-Pérot filter [7]. $\alpha$ was measured to be -0.06 at $6 \mathrm{GHz}$, a value 13 time smaller than the -0.77 chirp parameter of the standard Z-cut device designed with the same electrode length. The experimental data are marked with a cross in Figs. 2 and 3 and can be compared with the experimental data relative to the standard modulator, which are marked with circles. This confirm the theoretical predictions in the frequency range of concern.

It should be noted that practical applications typically require radio-frequency drive voltage around 5-6 V, which trans- lates to about 4-5 $\mathrm{V}$ at dc. As such, a length of about $3 \mathrm{~cm}$ would be needed. This length would lead to a 40-GHz chirp parameter $\alpha=-0.19$ according to (5) and (6), and taking $L_{1}=L_{2}=1.5 \mathrm{~cm}$, and a typical electrical loss coefficient $a_{0}=0.02 \mathrm{~dB} / \mathrm{cm} / \mathrm{GHz}^{1 / 2}$, a value which is still far beyond the chirp parameter of standard structure. So, the presented structure applies with telecommunication requirements, even in case of longer electrodes.

\section{CONCLUSION}

Ferroelectric domain inversion associated with electrode inversion has been proposed to control and adjust the chirp parameter of a Z-cut $\mathrm{LiNbO}_{3}$ modulator. The chirp parameter can be set to a given value by adjusting the ratio $L_{1} / L_{2}$ between the length of the noninverted $\left(L_{1}\right)$ and inverted $\left(L_{2}\right)$ ferroelectric sections. In the example discussed above, the chirp can be adjusted in a range between -0.8 and 0.8 . A zero-chirp Z-cut MZM was realized and tested up to $6 \mathrm{GHz}$, yielding a 6.6 half-wave voltage, a $-14 \mathrm{~dB}$ extinction ratio, and a -0.06 parameter. Work is in progress to fabricate modulators operating beyond $10 \mathrm{GHz}$.

\section{ACKNOWLEDGMENT}

The authors would like to thank J. Vendeville, D. Bitschène, and J. C. Renaud for technical assistance.

\section{REFERENCES}

[1] K. Hagimoto, M. Yoneyama, A. Sano, A. Hirano, T. Katacka, T. Otsuyi, K. Sato, and K. Noguchi, "Limitations and challenges of single carrier full $40 \mathrm{Gbit} / \mathrm{s}$ repeater system based on system equalization and new circuit design," in Proc. OFC'97, 1997, Paper ThC1, pp. 242-243.

[2] K. Yonenaga, Y. Miyamoto, H. Toba, K. Murata, M. Yoneyama, Y. Yamane, and H. Miyazawa, "320 Gbits/s WDM repeaterless transmission using fully encoded $40 \mathrm{Gbit} / \mathrm{s}$ optical duobinary channels with dispersion tolerance of 380 ps/nm," Electron. Lett., vol. 37, pp. 109-110, Jan. 2001.

[3] M. M. Howertob, R. P. Moeller, A. S. Greenblatt, and R. Krähenbühl, "Fully packaged, broadband $\mathrm{LiNbO}_{3}$ modulator with low drive voltage," IEEE Photon. Technol. Lett., vol. 12, pp. 792-794, July 2000.

[4] P. Jiang and A. C. O'Donnell, "LiNbO ${ }_{3}$ Mach-Zehnder modulators with fixed negative chirp," IEEE Photon. Technol. Lett., vol. 8, pp. 1319-1321, Oct. 1996.

[5] S. K. Korotky, J. J. Veselka, C. T. Kemmerer, W. J. Minford, D. T. Moser, J. E. Watson, C. A. Mattoe, and P. L. Stoddard, "High-speed low power optical modulator with adjustable chirp parameter," presented at the Integrated Photon. Res. Conf., Monterey, CA, 1991, Paper TuG2.

[6] Koyama and K. Iga, "Frequency chirping in external modulators," $J$. Lightwave Technol., vol. 6, pp. 87-93, Jan. 1988.

[7] T. Kawanishi, K. Kogo, S. Oisawa, and M. Izutsu, "Direct measurement of chirp parameters of high speed Mach-Zehnder type optical modulators," Opt. Commun., vol. 195, pp. 399-404, Aug. 2001. 